\title{
Analytical Solution of Nonlinear Diffusion Equation in Two-Dimensional Surface
}

\author{
A.J. JANAVIČIUS ${ }^{a, *}$ AND S. TURSKIENE $\dot{E}^{b}$ \\ ${ }^{a}$ Faculty of Natural Sciences, Šiauliai University \\ Višinskio 25, Šiauliai, 76351, Lithuania \\ ${ }^{b}$ Faculty of Mathematics and Informatics, Šiauliai University \\ Višinskio 19, Šiauliai, 76351, Lithuania
}

(Received July 4, 2005)

\begin{abstract}
We considered practically important case of nonlinear diffusion in the anisotropic plane where diffusion coefficients for diffusion in $x$ and $y$ axis directions can be different. This equation was transformed using similarity variables. The approximate analytical solution of the transformed equation expressed by power-series expansion for two variables about the zero point including only the first terms. The graphic representations show sufficient accuracy of the obtained analytical solution.
\end{abstract}

PACS numbers: 66.30.-h

\section{Introduction}

The classical linear diffusion equation is derived from the Fokker-Planck equation. This linear diffusion equation is obtained by assuming that process is slow. We see that fitting an experimental profile tail region of impurities to classical solution $\operatorname{erfc}(x / 2 \sqrt{D t})$ tail region, where linear diffusion must occur with large velocity, is physically non acceptable and cannot be fitted with experiment $[1,2]$. Any diffusion theory and equation must be conformed with the Brownian motion from where it follows that the diffusion velocity and maximum penetration depth of impurity atoms must be finite [3-5]. The diffusion coefficient must be equal to zero in the region where the impurities are absent. According to this realistic assumption the following nonlinear diffusion equation was proposed [1]:

$$
\frac{\mathrm{d}}{\mathrm{d} t} N=\frac{\mathrm{d}}{\mathrm{d} x}\left(D(N) \frac{\mathrm{d}}{\mathrm{d} x} N\right)
$$

where the nonlinear diffusion coefficient $D(N)$ was directly proportional to the

*corresponding author; e-mail: AYanavi@takas.lt 
impurities concentration $N[1,2]$ :

$$
D(N)=\frac{1}{N_{\mathrm{S}}} D N
$$

where $D$ is the diffusion coefficient for linear equation and $N_{\mathrm{S}}$ is the impurities concentration at the source of impurities. From the above presented equations and [4] we can obtain the nonlinear diffusion equation in two-dimensional case

$$
\begin{aligned}
& \frac{\mathrm{d}}{\mathrm{d} t} N=\frac{\mathrm{d}}{\mathrm{d} x}\left(D_{1}(N) \frac{\mathrm{d}}{\mathrm{d} x} N\right)+\frac{\mathrm{d}}{\mathrm{d} y}\left(D_{2}(N) \frac{\mathrm{d}}{\mathrm{d} y} N\right), \\
& D_{1}(N)=\frac{1}{N_{\mathrm{S}}} D_{1},
\end{aligned}
$$

with different linear diffusion coefficients $D_{1}$ and $D_{2}$ on $x$ and $y$ axis when off-diagonal elements are equal to zero [6]. In this case jumps of diffusing particles can occur in plane on $x$ and $y$ axis and in orthogonal directions. It can be surfaces of orthorhombic crystals [6].

\section{The analytical solution of the nonlinear two-dimensional diffusion equation}

Introducing the similarity variables in (1.3)

$$
\xi_{1}^{\prime}=\xi_{1}-\xi_{10}, \quad \xi_{2}^{\prime}=\xi_{2}-\xi_{20}, \quad \xi_{1}=\frac{x}{\sqrt{D_{1} t}}, \quad \xi_{2}=\frac{y}{\sqrt{D_{2} t}}
$$

and new function

$$
N(x, y, t)=N_{\mathrm{S}} f\left(\xi_{1}^{\prime}, \xi_{2}\right)
$$

we obtain the following nonlinear differential equation:

$$
2 \frac{\mathrm{d}}{\mathrm{d} \xi_{1}^{\prime}}\left(f \frac{\mathrm{d} f}{\mathrm{~d} \xi_{1}^{\prime}}\right)+2 \frac{\mathrm{d}}{\mathrm{d} \xi_{2}^{\prime}}\left(f \frac{\mathrm{d} f}{\mathrm{~d} \xi_{2}^{\prime}}\right)+\xi_{1}^{\prime} \frac{\mathrm{d} f}{\mathrm{~d} \xi_{1}^{\prime}}+\xi_{2}^{\prime} \frac{\mathrm{d} f}{\mathrm{~d} \xi_{2}^{\prime}}+\xi_{10} \frac{\mathrm{d} f}{\mathrm{~d} \xi_{1}^{\prime}}+\xi_{20} \frac{\mathrm{d} f}{\mathrm{~d} \xi_{2}^{\prime}}=0 .
$$

Here $\xi_{10}$ and $\xi_{20}$ represents the maximum penetration points of impurities on axis $x$ and $y$. The approximate solution of the obtained equation can be expressed by power expansion

$$
f\left(\xi_{1}^{\prime}, \xi_{2}^{\prime}\right)=a_{1} \xi_{1}^{\prime}+a_{2} \xi_{2}^{\prime}+a_{3} \xi_{1}^{\prime 2}+a_{4} \xi_{2}^{\prime 2}+a_{5} \xi_{1}^{\prime} \xi_{2}^{\prime}
$$

where terms are included till second power [7]. Taking into account that Eq. (2.3) is symmetrical in variables $\xi_{1}^{\prime}, \xi_{2}^{\prime}$ and requiring the same symmetry for solution (2.4) we obtained

$$
a_{1}=a_{2}, \quad a_{3}=a_{4} .
$$

We use boundary conditions

$$
\begin{aligned}
& f\left(-\xi_{10},-\xi_{20}\right)=1, \\
& f\left(0,-\xi_{20}\right)=0, \quad f\left(-\xi_{10}, 0\right)=0
\end{aligned}
$$

requiring that we have invariable concentration (2.2) of impurities $N_{\mathrm{S}}$ in the center of coordinates and that impurities are spreading with finite velocity attaining points $\xi_{10}$ and $\xi_{20}$. 
Substituting expression (2.4) in Eq. (2.3), equating the coefficient till first power of variables to zero and including conditions $(2.5),(2.6),(2.7)$ we obtain

$$
\begin{aligned}
& 4 a_{1}+\xi_{10}+\xi_{20}=0, \\
& 16 a_{1} a_{3}+4 a_{1} a_{5}+a_{1}+2 a_{3} \xi_{10}+a_{5} \xi_{20}=0, \\
& -a_{1} \xi_{10}-a_{1} \xi_{20}+a_{3}\left(\xi_{10}\right)^{2}+a_{3}\left(\xi_{20}\right)^{2}+a_{5} \xi_{10} \xi_{20}=1, \\
& -a_{1}+a_{3} \xi_{10}=0, \\
& -a_{1}+a_{3} \xi_{20}=0 .
\end{aligned}
$$

Solving the system of five Eqs. (2.8), we can find the five unknown coefficients

$$
a_{1}=a_{2}=-0.316, \quad a_{3}=a_{4}=-0.500, \quad a_{5}=2.50, \quad \xi_{10}=\xi_{20}=0.632 .
$$

\section{Conclusions}

The obtained solution (2.2), (2.4), with coefficients (2.9) of nonlinear diffusion equation (1.3) can be used for theoretical calculations of impurities spreading by diffusion from constant sources in semiconductors films. Our results can be used for the heat transport problem in surfaces of materials heated with lasers [8], because diffusion equation has the same form like heat transport equation. It is interesting to note that for nonlinear diffusion for nonisothermical case we obtained the same inertia phenomena for diffusion of impurities [9] like thermal conductivity inertia in thin gold film heated with ultrashort laser pulse [8]. Using method proposed in paper [9] we can easily transform our solution for diffusion and heat transport in nonisothermical case. For this aim diffusion or heat conductivity coefficients must be divided by excitation parameter [9]. The obtained solution can also be approximately used when we have diffusion of ionized gases in the isotropy

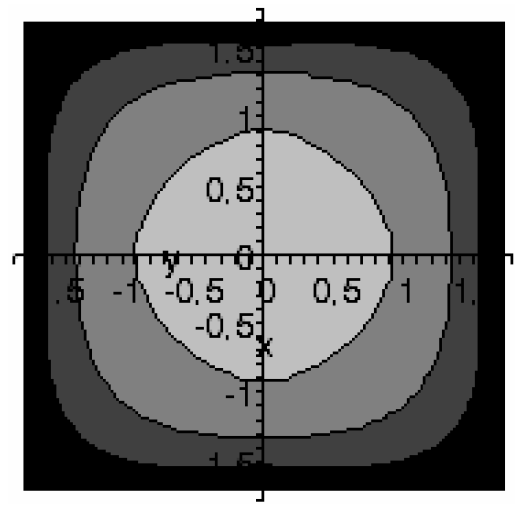

Fig. 1. Contour plots in plane when $D_{1}=D_{2}$. 


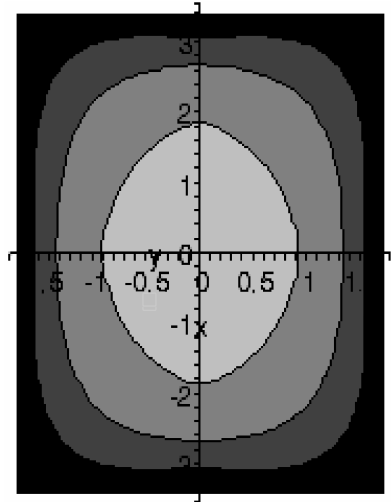

Fig. 2. Contour plots in plane when $D_{2}=4 D_{1}$.

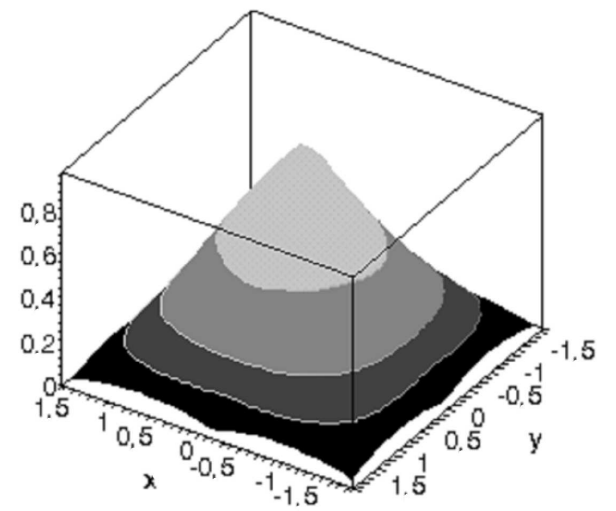

Fig. 3. Space contour plots when $D_{1}=D_{2}$.

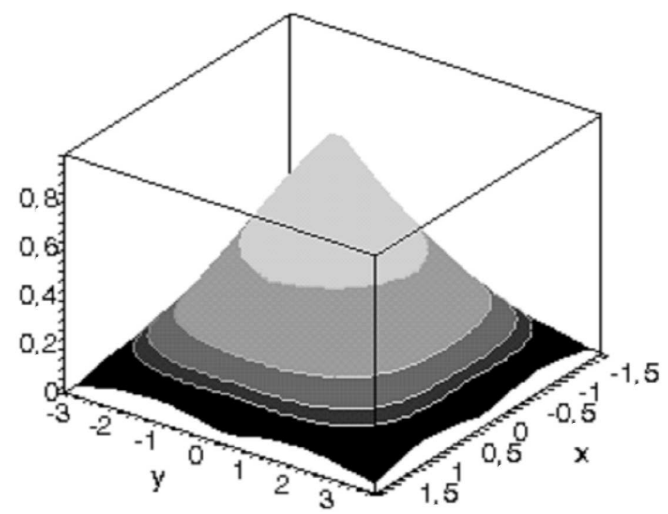

Fig. 4. Space contour plots when $D_{2}=4 D_{1}$. 
medium when weak electrical field acts in definite direction. The relative concentrations of diffusing impurities are presented in the plane of Fig. 1 and Fig. 2 and in the space representations Fig. 3 and Fig. 4 for the cases $D_{1}=D_{2}$ and $D_{2}=4 D_{1}$, consequently. For example in our calculations we used $D_{1}=0.182 \mathrm{~cm}^{2} / \mathrm{s}$. From the presented figures we see that our solution is only approximate.

\section{References}

[1] A.J. Janavičius, V. Stukaitè, D.J. Zanevičius, Electron Tech. Ser. 2, Semicond. Dev. 160, 27 (1983) (in Russian).

[2] Way-Seen Wang, Yu-Hwa Lo, IEEE Trans. Electron Dev. ED-30, 1828 (1983).

[3] A.J. Janavičius, Phys. Lett. A 224, 159 (1997).

[4] A.J. Janavičius, G. Lūža, D. Jurgaitis, Acta Phys. Pol. A 105, 475 (2004).

[5] A.J. Janavičius, A. Poškus, Acta Phys. Pol. A 105, 519 (2005).

[6] Martin Eden Glicksman, Diffusion in Solids Field Theory, Solid-State Principles, and Applications, Wiley, New York 2000, p. 472.

[7] J. Janavičius, Ž. Norgèla, J. Jurgaitis, Math. Model. Anal. 6, 77 (2001).

[8] J. Marciak-Kozlowska, Z. Mucha, Lith. J. Phys. 35, 616 (1995).

[9] A.J. Janavičius, Acta Phys. Pol. A 93, 505 (1998). 